\title{
The Case for Neurological Registry Best Practice Guidelines in Canada
}

\author{
Nathalie Jette $^{1,2}$, Megan Johnston ${ }^{1}$, Tamara Pringsheim ${ }^{1}$, Lawrence Korngut ${ }^{1}$
}

Can J Neurol Sci. 2013; 40: Suppl. 2 - S1-S3

\section{RATiONALE}

\section{Why are neurological conditions so important to Canadians?}

In 2005, The World Health Organization (WHO) reported that neurological conditions account for over $6 \%$ of the global burden of disease. ${ }^{1}$ The relative contribution of neurological conditions is greater in high income countries such as Canada. ${ }^{1}$ The burden of neurological conditions is substantial because many: (1) are chronic and lack curative therapies; (2) occur or manifest throughout the lifespan (e.g. epilepsy, traumatic brain injury); (3) follow a progressive course; (4) lead to functional limitations; and (5) require significant healthcare resources and caregiver investment. The WHO predicts that the healthcare burden from neurological conditions will increase over the next 20 years. Estimated total deaths attributed to neurological conditions are predicted to rise by approximately $0.6 \%$ by 2030 while estimated total disability is predicted to rise by about $0.5 \%{ }^{1}$

A recent report from the Canadian Institute for Health Information (CIHI) focusing on 11 neurological conditions reported that in Canada: (1) the total cost of these conditions ( $\$ 8.8$ billion) represented $7 \%$ of the total attributable cost of all illness while nine of the 11 conditions accounted for $8.3 \%$ of the total indirect cost of illness ( $\$ 6.5$ billion per year) in 2000-2001; (2) six of the 11 conditions accounted for $10.6 \%$ of the total disability adjusted life years in Canada; (3) in 2004-2005, nearly $20 \%$ of patient days in Canadian acute care hospitals were for persons affected by one of the 11 conditions and (4) in 2005$2006,50 \%$ of complex continuing care stays were for patients with Alzheimer's disease (AD), amyotrophic lateral sclerosis (ALS), cerebral palsy (CP), epilepsy, traumatic brain injury (TBI), multiple sclerosis (MS), Parkinson's disease (PD) or stroke. $^{2}$

\section{National Population Health Study of Neurological Conditions}

On June 5, 2009 the federal Minister of Health announced the four year National Population Health Study of Neurological Conditions. ${ }^{3}$ This study was led by the Public Health Agency of Canada in collaboration with Neurological Health Charities Canada; the Canadian Institutes of Health Research and Health Canada. The objectives of the study were to improve knowledge of the scope of 14 neurological conditions in Canada (incidence, prevalence, and co-morbidities); use of health services; gaps in services and recommended improvements; and impacts of neurological conditions now and projected over the next 20 years (including economic cost). A comprehensive report of the study findings will be published in 2014 .

\section{Information on the burden of neurological conditions is} limited or unavailable

The WHO's Global Burden of Disease report (2006) and CIHI's report on the Burden of Neurological Diseases, Disorders, and Injuries in Canada (2007) both found that while the burden of neurological disorders in Canada is high (over $10 \%$ of total disability), complete information on the burden of these conditions is unavailable. ${ }^{1,2}$ Indeed, for CIHI's report, only 6 out of 11 neurological conditions examined had estimates of disability burden.

\section{Patient registries are a key source of data to assess the burden of neurological conditions}

A patient registry can be defined as an observational cohort study of real-world clinical practice related to a disease condition or procedure/therapy, without a study-mandated treatment. With the ability to securely catalogue and track many patients across large geographical areas, registries can provide epidemiological data and fill gaps in medical evidence. ${ }^{4}$ In addition to tracking disease burden and therapeutic effectiveness, registries may be useful for tracking the use of medical therapies, performance measurement for the purpose of quality improvement, evaluating the "real world" effectiveness of medical therapies in practice outside the highly controlled conditions of clinical trials, identifying relationships between risk factors and disease outcomes, and evaluating access to care. Despite the utility of registries little guidance is available for investigators and stakeholders on the quality of information derived from these data sources. ${ }^{4}$

The WHO's World Health Report identified five core competencies for long term patient care. ${ }^{1}$ One of these five core competencies was the development of information and communication technologies including registries to ensure continuity of care.

From the ${ }^{1}$ Hotchkiss Brain Institute, University of Calgary, Calgary, Alberta; ${ }^{2}$ Institute for Public Health, University of Calgary, Calgary, Alberta.

Final Revisions Submitted January 28, 2013.

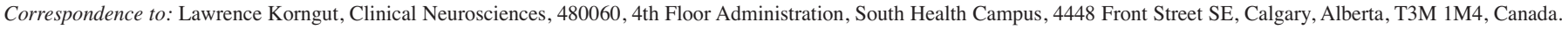

Email: Lawrence.korngut@albertahealthservices.ca. 
As Canada's healthcare system faces multiple stressors over the next 20 years including increasing costs, mismatches between human resource supply and demand, and an aging population, solutions to address the increasing burden of neurological disease must be identified urgently. The first step towards this goal is to improve the available information on neurological disease burden in Canada.

The key to successful national neurological registries is to develop consensus guidelines and a toolkit that will guide registry leaders in their development to ensure comprehensive, systematic, and meaningful collection of data.

Patient registries present an important opportunity to improve the information available in Canada on neurological disease burden. However, statistics collected from patient registries will only be meaningful if the registries are implemented consistently so the data collected can be compiled and compared.

A significant need exists for comprehensive guidelines for registry development (including online registries) and implementation within neurological disease in Canada. Varying provincial privacy regulations and research ethics review board (REB) perceptions result in logistical and financial obstacles to multi-regional and national registry implementation and operation within Canada. The development of consensus guidelines targeting best practices and identified obstacles will facilitate current registry operations and the design and implementation of new registries.

\section{Methods}

To inform the guideline development process we performed a comprehensive exploratory literature review. Patient and caregiver focus groups were concomitantly performed to ensure the relevance of the guidelines to the target population.

\section{Literature Review}

A literature review aiming to identify all patient registryrelated literature was performed using search terms such as register, registry and registries. The search strategy (see Appendix A) was developed in consultation with a research librarian and included the following databases: Medline, EMBASE, Pubmed, Cochrane Central, Cochrane SR, PsycINFO, ABI Inform, BIOSIS Previews, and PAIS (Public Affairs Information Service). Figure 1 outlines the flow of article identification and screening. We identified 19,002 abstracts with 6,435 remaining after duplicates and non-English articles were removed. The first reviewer excluded 2, 238 abstracts. Included abstracts were then reviewed by a second reviewer and a further 3,787 abstracts were excluded. In total 410 full-text articles were reviewed. Relevant aspects of this literature review are outlined in this document and served to inform the guideline development process.

\section{Guideline Development}

This guideline document was developed through an iterative process involving multiple stakeholders. In April of 2012, three patient and caregiver focus groups were held at the University of Calgary. The methods and results of the focus groups are published elsewhere.
Second, the results of the literature review and the focus groups were presented at a preliminary meeting of registry and disease experts held in Calgary in May 2012. At this meeting, the overall climate for registry development in Canada was discussed and work teams were formed to brainstorm and develop the sections of this document. Finally, a second meeting was held in Calgary in September 2012 with work team members, and additional registry, disease experts, and other stakeholders (e.g. ethics, legal, privacy) to finalize the content of this guidelines document.

\section{Toolkit Development}

Accompanying this guideline document is a toolkit of resources conceived to assist in the design and implementation of new neurological registries in Canada. This toolkit was assembled from a variety of existing resources across the entire registry spectrum. The toolkit and the guideline document are intended to be used in concert and it is our sincere hope that it will be a helpful resource. The complete toolkit is available at http://www.canadianregistrynetwork.org.

\section{Overview}

This guideline document is organized into three parts consecutively addressing registry design, quality and impact. Each part begins with an executive summary that summarizes key points. More in-depth and supporting information is presented thereafter.

It is our hope that this guideline document and accompanying toolkit will be useful to registry leaders, staff, investigators, patient organizations, governmental agencies, the pharmaceutical and biotechnology industries, and other institutions, groups and individuals with respect to the following:

1. Determining whether a registry is appropriate to address a specific question or series of questions

2. Providing resources to assist in developing the case for a registry

3. Providing a comprehensive framework for registry design (i.e. protocol development, ethics board submission, data collection infrastructure development)

4. Understanding and addressing the importance of quality control and assurance

5. Techniques in validation and interpretation of registry data

6. The importance of the impact of a registry and its measurement 


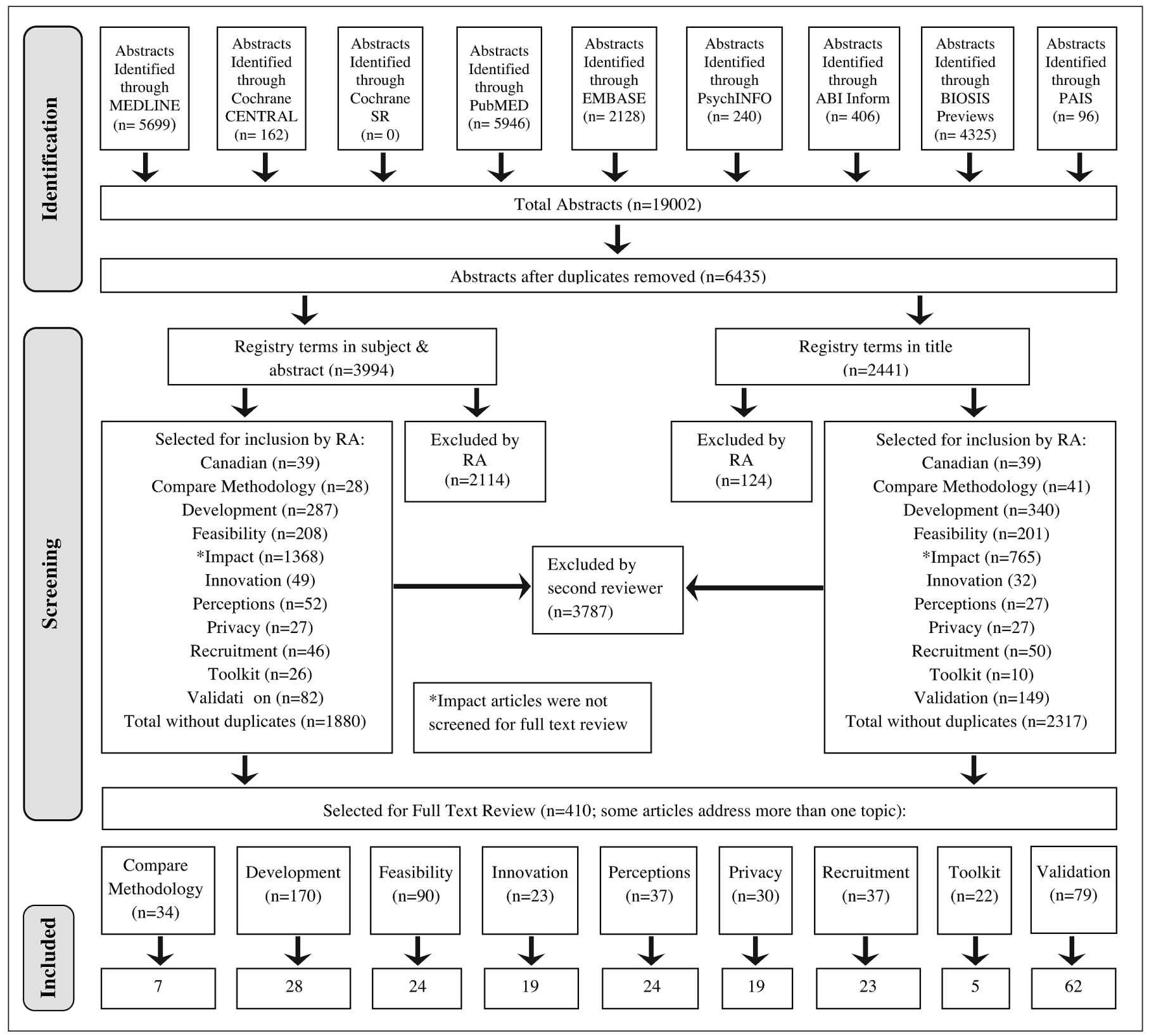

Figure 1: Registry Literature Review Flowchart. Outline of the flow of article identification and selection.

This guideline document and accompanying toolkit can also be used to:

1. Identify appropriate references from the literature to support funding application and manuscript preparation

2. Support registry standards and best practices in Canada in funding applications and ethics board submissions.

3. Provide published benchmarks for data quality

4. Provide examples of registry impact

A key additional resource to which all users of this document may wish to refer is the Agency for Healthcare Research and
Quality "Registries for Evaluating Patient Outcomes: A User's Guide" document (AHRQ manual). ${ }^{5}$ Throughout this guideline document we have highlighted specific areas where the AHRQ manual is relevant and useful in the Canadian context. During the preparation of this guideline we utilized the Second Edition of the AHRQ manual (http://www.innovations.ahrq.gov/content. aspx?id=3012). A new edition is forthcoming in 2013. 